\title{
INFECCIONES RESPIRATORIAS AGUDAS Y CARACTERIZACIÓN DE BACTERIAS POTENCIALMENTE PATÓGENAS EN COMUNIDADES DE LA HUASTECA POTOSINA.
}

ACUTE RESPIRATORY INFECTIONS AND CHARACTERIZATION OF POTENTIALLY PATHOGENIC BACTERIA IN COMMUNITIES OF LA HUASTECA POTOSINA.

Diego-Rodríguez Mariana*, Domínguez-Cortinas Gabriela*, Cubillas-Tejeda Ana Cristina*, Galindo Mendoza María Guadalupe*.

*Universidad Autónoma de San Luis Potosí, México.

Citation: Diego-Rodríguez M., Domínguez-Cortinas G., Cubillas-Tejeda A.C., Galindo Mendoza M.G. (2019) Infecciones respiratorias agudas y caracterización de bacterias potencialmente patógenas en comunidades de la Huasteca Potosina. Revista Salud Pública y Nutrición, 18 (4), 1-8.

Editor: Esteban G. Ramos Peña, Dr. CS., Universidad Autónoma de Nuevo León, Facultad de Salud Pública y Nutrición, Monterrey Nuevo León, México. Copyright: (C2019 Diego-Rodríguez M. et al. This is an open-access article distributed under the terms of Creative Commons Attribution License [CC BY 4.0], which permits unrestricted use, distribution, and reproduction in any medium, provided the original author and source are credited.

Competing interests: The authors have declared that no competing interests exist.

DOI: https://doi.org/10.29105/respyn18.4-1

Recibido: 02 de agosto 2019; $\quad$ Aceptado: 26 de noviembre 2019

Email: gabriela.dominguez@uaslp.com 


\title{
INFECCIONES RESPIRATORIAS AGUDAS Y CARACTERIZACIÓN DE BACTERIAS POTENCIALMENTE PATÓGENAS EN COMUNIDADES DE LA HUASTECA POTOSINA.
}

\author{
Diego-Rodríguez Mariana*, Domínguez-Cortinas Gabriela*, Cubillas-Tejeda Ana Cristina*, Galindo Mendoza \\ María Guadalupe*. \\ *Universidad Autónoma de San Luis Potosí, México.
}

\section{RESUMEN}

Introducción. Las enfermedades respiratorias generan una inmensa carga para la salud en todo el mundo, entre ellas se encuentra la enfermedad pulmonar obstructiva crónica, la tuberculosis, asma e infecciones respiratorias agudas. Estas últimas son responsables de más de 4 millones de muertes cada año. Objetivo: Conocer tanto la prevalencia de infecciones respiratorias agudas como la frecuencia de bacterias potencialmente patógenas en garganta de población infantil y adulta, así como algunos factores de riesgo asociados en comunidades vulnerables del estado de San Luis Potosí. Material y Método: Se seleccionaron 3 localidades del estado de San Luis Potosí (105 familias). Se realizaron exudados faríngeos para identificar bacterias potencialmente patógenas (BPP) y por medio de cédulas familiares se obtuvieron datos de síntomas de infecciones respiratorias agudas un mes antes de la entrevista y algunos factores de riesgo. Resultados Se lograron identificar BPP en las tres localidades, encontrándose con mayor frecuencia a Enterobacter spp (34.6\% en el Sabinito), Escherichia coli (40\% en Nueva Primavera), Streptococcus pyogenes (37.5\% en Nuevo Aquismón). Conclusiones: Los resultados encontrados son un primer acercamiento de un análisis epidemiológico de BPP en localidades que presentan vulnerabilidad socioeconómica y de salud.

Palabras Clave: Infecciones respiratorias agudas, bacterias patógenas potenciales, factores de riesgo.

\section{ABSTRACT}

Introduction: Respiratory diseases generate an immense burden for health worldwide, including chronic obstructive pulmonary disease, tuberculosis, asthma and acute respiratory infections. The latter are responsible for more than 4 million deaths each year. Objective: Know both the prevalence of acute respiratory infections and the frequency of potentially pathogenic bacteria in the throat of children and adults, as well as some associated risk factors in vulnerable communities in the state of San Luis Potosí. Material and method: Three locations in the state of San Luis Potosí (105 families) were selected. Pharyngeal exudates were performed to identify potentially pathogenic bacteria (BPP) and data from acute respiratory infections were obtained one month before the interview and some risk factors. Results: They were able to identify BPP in the three locations, with Enterobacter spp ( $34.6 \%$ in Sabinito), Escherichia coli ( $40 \%$ in New Spring), Streptococcus pyogenes (37.5\% in Nuevo Aquismón) being more frequently found. Conclusions: The results found are a first approach to an epidemiological analysis of BPP in localities that present socioeconomic and health vulnerability.

Key words: Acute respiratory infections, potential pathogenic bacteria, risk factors. 


\section{Introducción}

Las infecciones respiratorias agudas (IRA) se definen como el conjunto de enfermedades transmisibles del aparato respiratorio que incluye desde el catarro común hasta la neumonía, pasando por la otitis, amigdalitis, sinusitis, bronquitis aguda, laringotraqueitis, bronquiolitis y laringitis, con evolución menor a 15 días y con la presencia de uno o más síntomas o signos clínicos como tos, rinorrea, obstrucción nasal, etc., los cuales pueden estar o no acompañados de fiebre (Ferreira, et al., 2013). Son generalmente de etiología infecciosa, pueden producir un espectro de enfermedades que van desde infecciones asintomáticas o leves hasta enfermedades graves y fatales, dependiendo del patógeno causante, de factores ambientales y del huésped (Secretaría de Salud [SSA], 2012) (Tapia, Sarti, Kuri, Ruíz, Velázquez, 2006).

Si bien es cierto que la mayoría de las infecciones respiratorias tienen un origen viral, las bacterias juegan un papel importante en la colonización del epitelio respiratorio superior que posteriormente si alcanza el tracto respiratorio inferior ocasiona un grupo complejo y extenso de enfermedades que de no ser tratadas a tiempo pueden generar complicaciones graves sobre todo en grupos etarios vulnerables como son los niños y adultos mayores (Somogyi, Alfaro, Herrera, Herrera, 1998).

De acuerdo con la organización mundial de la salud (OMS) las IRA continúan siendo la enfermedad transmisible más letal, tan sólo en 2016 causaron tres millones de defunciones en todo el mundo (Organización Mundial de la Salud [OMS], 2018). En niños menores de 5 años, la neumonía es responsable del $18 \%$ de todas las muertes o más de 1,3 millones al año (Walker, et al.,2013); la neumonía mata a muchas más personas que el VIH o el paludismo (Neil, 2010). De acuerdo con las estadísticas oficiales, en México las IRA constituyen la primera causa de morbilidad y egreso hospitalario en los menores de 5 años, con frecuencia de 13 episodios por cada 100 consultas médicas. Lo anterior representa dos a cuatro episodios de IRA al año por niño (Dirección General de Epidemiología [DGE], 2012).

Para el estado de San Luis Potosí las IRA siguen siendo relevantes; de acuerdo con la Encuesta Nacional de Salud y Nutrición identificó una prevalencia en niños menores de 10 años del $38.0 \%$ apenas por debajo de la prevalencia Nacional con un 40.0\% (Encuesta Nacional de Salud y Nutrición [ENSANUT], 2012).

También cabe mencionar que resulta muy importante el papel que juega la flora bacteriana común o normal del tracto respiratorio humano, además la nasofaringe humana constituye un reservorio natural de bacterias potencialmente patógenas (BPP), que se involucran en varios procesos infecciosos, sobre todo, en la población infantil (Lieberman, et al., 2006) (Neto, et al., 2003).

Entre las principales BPP que causan infección ya sea respiratoria o meníngea se señalan: Staphylococcus aureus, Haemophilus influenzae, Moraxella catarrhalis, Streptococcus pneumoniae y Streptococcus pyogenes, además la Neisseria meningitidis que puede causar meningitis y/o enfermedad meningocóccica (Álvarez, Roas, Maitín, 2008). Estas bacterias colonizan la nasofaringe de los individuos sanos y contribuyen al desarrollo de infecciones locales o invasivas. Su presencia en la nasofaringe origina el estado de portador sano, persona que alberga un agente infeccioso sin presentar signos o síntomas clínicos de enfermedad y que puede constituir una fuente potencial de infección (Fuentes, et al., 2002).

Los factores de riesgo que favorecen la colonización nasofaríngea por las BPP y el estado del portador son varios y entre ellos se describen: la edad, el sexo, el hábito de fumar, condición de fumador pasivo, ausencia de lactancia materna, el consumo de bebidas alcohólicas, el hacinamiento, la ocupación, las infecciones respiratorias aguda (IRA), el tratamiento con esteroides, antimicrobianos o drogas inmunosupresoras, así como los antecedentes alérgicos (asma bronquial), entre otros (Neto, et al., 2003) (Castellanos, et al., 2001).

El presente estudio tuvo por objetivo conocer la prevalencia de infecciones respiratorias agudas y detectar la presencia de bacterias potencialmente patógenas en garganta de población infantil y adulta, así como algunos factores de riesgo asociados a estas en tres comunidades de la Huasteca Potosina. 
Consideraciones éticas

Toda la metodología se apegó estrictamente a la declaratoria de ética de Helsinki. La participación de fue voluntaria, todos firmaron una carta de consentimiento informado, en el caso de los menores de edad este consentimiento fue firmado por un padre o tutor, con la conformidad del menor. Cada resultado de los estudios realizados se entregó en forma confidencial.

\section{Material y Método}

Selección de comunidades de estudio

Se aplicaron los siguientes criterios para la selección de comunidades de estudio: a) Localidades con población entre 500 y 1200 habitantes, b) Grado de marginación y rezago social alto-medio y d) Ubicadas en microrregiones geográficas con señales definidas de variabilidad climática (DomínguezCortinas, 2013). Siguiendo los criterios anteriormente mencionados se eligieron las comunidades: Nueva Primavera (Municipio Tamuín), Nuevo Aquismón (Municipio Tamuín) y El sabinito de Arriba (Municipio El Naranjo). Estas localidades forman parte de la Huasteca potosina que se localiza en el extremo sur oriental del estado de San Luis Potosí.

\section{Diseño y tipo de estudio}

El presente estudio fue de tipo exploratorio, descriptivo, con diseño de campo y de corte transversal. El periodo de evaluación para analizar las IRA y la detección de BPP en cada comunidad fue de enero a marzo del 2017.

Muestreo y población de estudio

La población de estudio de cada comunidad fue elegida según los siguientes criterios de inclusión: a) Tiempo de residencia en la comunidad de10 años o más para población adulta y de toda la vida para población infantil, b) Aceptación para participar en el estudio mediante una carta de consentimiento informado y c) Adultos y niños que no estuvieran bajo tratamiento de alguna enfermedad respiratoria al momento de la encuesta. El total de población participante por comunidad quedó de la siguiente manera: El Sabinito $(\mathrm{N}=149)$, Nueva Primavera $(\mathrm{N}=108)$ y Nuevo Aquismón $(\mathrm{N}=235)$.

La obtención de información general del estado de salud, así como de otros datos socioeconómicos y demográficos de las poblaciones totales participantes, se llevó a cabo mediante una encuesta semiestructurada aplicada por unidad familiar y respondida por el jefe o jefa de familia. Los datos que se recogieron fueron: 1) Población infantil con diagnóstico de IRA que se definió como porcentaje de la población de 15 años o menor que recibió un diagnóstico médico de IRA por un mes antes del periodo de evaluación. 2) Población adulta mayor de 15 años con diagnóstico de IRA que se definió igual que el indicador anterior 3) Algunos factores de riesgo de interés (edad y hacinamiento).

Para la obtención de las BPP se realizaron 316 exudados faríngeos a niños y adultos seleccionados aleatoriamente de la población total participante de cada comunidad garantizando en cada caso una representatividad mayor del 40\% (El Sabinito $n=120$; Nuevo Aquismón n=153; Nueva Primavera n=43).

\section{Exudados Faríngeos}

Los exudados faríngeos fueron realizados frotando áreas amigdalares con hisopo de algodón estéril, colocado inmediatamente en medio de transporte Stuart para evitar contaminación y conservados en hielera a $4^{\circ} \mathrm{C}$ para su transporte al laboratorio. Las muestras se inocularon en Agar Mueller-Hinton (Merck) suplementado con sangre de carnero desfibrinada al 5\% (para el aislamiento de S. aureus, Streptococcus spp). Las placas inoculadas se estriaron e incubaron a $37^{\circ} \mathrm{C}$ durante 24 a $48 \mathrm{~h}$ en atmósfera húmeda con $5 \%$ de $\mathrm{CO} 2$. Luego de su incubación, se re aislaron los microorganismos que pudieran corresponder con S. aureus, Estreptococo $\beta$-hemolítico, S. pneumoniae. Se investigaron también por los métodos convencionales (Koneman, et al., 1998) otras BPP identificadas por tinción de Gram, como bacilos gramnegativos (BGN). Entre ellos se aislaron cepas de Klebsiella spp., Proteus spp. Y Pseudomonas spp.

\section{Control de calidad.}

Los análisis microbiológicos fueron realizados en el Laboratorio de Química Clínica/Análisis Clínicos de la Facultad de Ciencias Químicas, UASLP, el cual cuenta con certificación según NOM-007-SSA32011 y PROY-NMX-EC-17043-IMNC-2010.

\section{$\underline{\text { Análisis estadístico }}$}

Los resultados fueron procesados en bases de datos Excel 2013. Se realizaron pruebas de X2 $(p=<0.05)$ y Razón de Momios de Prevalencia (RMP) entre las 
BPP, factores de riesgo y localidades por medio del software Epi info 7.

\section{Resultados}

Bacterias potencialmente patógenas identificadas Entre las BPP identificadas predominó: Enterobacter spp. (34.6\% en el Sabinito), Escherichia coli (38.3\% en Nuevo Aquismón y $40 \%$ en Nueva Primavera), Streptococcus pyogenes $(37.5 \%$ en Nuevo Aquismón).

Las bacterias más frecuentes fueron Streptococcus pyogenes $(9.3 \%$ en el Sabinito, 37.5 en Nuevo Aquismón y 20\% en Nueva Primavera), Enterobacter spp (34.6\% en el Sabinito, 6.6\% en Nuevo Aquismón y $7 \%$ en Nueva Primavera), Escherichia coli (6.6\% en el Sabinito, $38.3 \%$ en Nuevo Aquismón y $40 \%$ en Nueva Primavera). (Tabla 1).

\begin{tabular}{|c|c|c|}
\hline Comunidad & Microorganismos & Frecuencias (\%) \\
\hline \multirow[t]{7}{*}{ El sabinito } & Enterobacter spp & $26(34.6)$ \\
\hline & Klebsiella spp. & $18(24)$ \\
\hline & Staphylococcus Aureus & $12(16)$ \\
\hline & Streptococcus Pyogenes & $7(9.3)$ \\
\hline & Escherichia coli & $5(6.6)$ \\
\hline & Otros $^{*}$ & $7(9.2)$ \\
\hline & Total & $75(100)$ \\
\hline \multirow[t]{7}{*}{ Nuevo Aquismón } & Escherichia coli & $46(38.3)$ \\
\hline & Streptococcus Pyogenes & $45(37.5)$ \\
\hline & Enterobacter spp & $8(6.6)$ \\
\hline & Klebsiella spp. & $6(5)$ \\
\hline & Streptococcus grupo $A$ & $5(4.1)$ \\
\hline & otros $^{* *}$ & $10(8.1)$ \\
\hline & Total & $120(100)$ \\
\hline \multirow[t]{6}{*}{ Nueva Primavera } & Escherichia coli & $6(40)$ \\
\hline & Klebsiella spp. & $4(26.6)$ \\
\hline & Streptococcus Pyogenes & $3(20)$ \\
\hline & Proteus spp. & $1(7)$ \\
\hline & Enterobacter spp. & $1(7)$ \\
\hline & Total & $15(100)$ \\
\hline
\end{tabular}

Prevalencias de infecciones respiratorias agudas (IRA)

a) Población infantil con diagnóstico previo de IRA.

La población infantil total con diagnóstico de IRA previo fue de 34 individuos (El Sabinito $n=12$, Nueva Primavera $\mathrm{n}=10$, Nuevo Aquismón $\mathrm{n}=12$ ). Se realizó análisis de $\mathrm{x} 2$ y RMP entre las tres localidades no mostrando ninguna diferencia estadísticamente significativa (Tabla 2). b) Población adulta mayor de 15 años con diagnóstico previo de IRA.

La población adulta total con diagnóstico de IRA fue de 69 individuos (El Sabinito $n=30$, Nueva Primavera $n=9$, Nuevo Aquismón $n=30$ ). Se realizó también análisis de $\mathrm{x} 2$ y RMP entre las tres localidades mostrando diferencia estadísticamente significativa $(\mathrm{p}=<0.00001)$ en la localidad del Sabinito. Además, la RMP de la misma comunidad fue de 4.1 (IC=2.08 - 10.07) (Tabla 2).

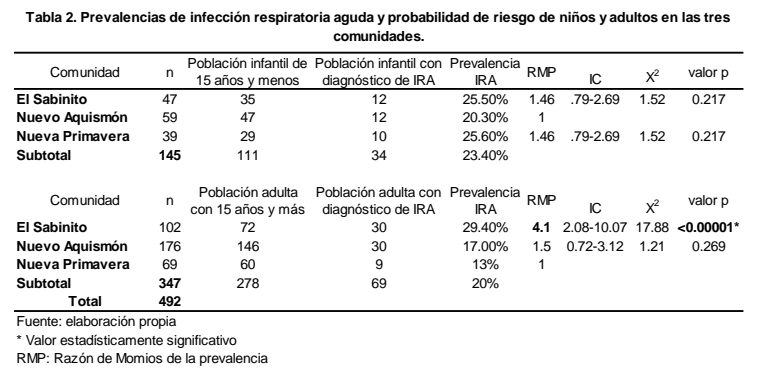

Portadores de bacterias potencialmente patógenas y factores de riesgo

Los resultados de los exudados faríngeos muestran que, en la comunidad de El Sabinito de 120 muestras analizadas, 70 resultaron con BPP dando una prevalencia del $58.30 \%$. Para la comunidad de Nuevo Aquismón, de 153 muestras, 105 mostraron BPP dando una prevalencia del $68.60 \%$ y para la comunidad de Nueva Primavera, de 43 muestras, 15 presentaron BPP con una prevalencia del $34.80 \%$. Esto es, un total de 190 portadores de BPP $(60 \%)$, considerando las tres comunidades de estudio (Tabla $3)$.

\begin{tabular}{|c|c|c|c|c|c|c|c|}
\hline \multicolumn{8}{|c|}{ Comunidad EI Sabinito } \\
\hline Edad (años) & $n$ & Portadores $\mathrm{n}(\%)$ & ) No portadores $\mathrm{n}(\%)$ & RMP & IC & $x^{2}$ & valor $p$ \\
\hline 1 a 15 & 46 & $32(70)$ & $14(30)$ & 2.52 & $1.41-4.5$ & 10.004 & $0.001^{\star}$ \\
\hline 16 a 30 & 24 & $12(50)$ & $12(50)$ & 1.083 & $0.62-1.88$ & 0.08 & 0.77 \\
\hline 31 a 55 & 33 & $16(48)$ & $17(52)$ & 1 & & & \\
\hline 56 a 80 & 17 & $10(59)$ & $7(41.1)$ & 1.55 & $0.89-2.72$ & 2.43 & 0.11 \\
\hline Total & 120 & 70 & 50 & & & & \\
\hline \multicolumn{8}{|c|}{ Comunidad Nuevo Aquismón } \\
\hline Edad (años) & $n$ & Portadores $\mathrm{n}(\%)$ & ) No portadores $\mathrm{n}(\%)$ & RMP & IC & $x^{2}$ & valor $p$ \\
\hline 1 a 15 & 37 & $30(81)$ & $7(19)$ & 1 & & & \\
\hline 16 a 30 & 39 & $19(49)$ & $20(51)$ & 0.22 & $0.11-0.42$ & 22.5 & $<0.00001^{\star}$ \\
\hline 31 a 55 & 45 & $36(80)$ & $9(20)$ & 0.9 & $0.46-1.88$ & 0.03 & 0.85 \\
\hline 56 a 80 & 26 & $16(62)$ & $10(38.4)$ & 0.38 & $0.20-0.72$ & 8.8 & $0.002^{\star}$ \\
\hline Más de 80 & 6 & $4(66.6)$ & $2(33.3)$ & 0.47 & $0.24-0.91$ & 5.09 & 0.024 \\
\hline Total & 153 & 105 & 48 & & & & \\
\hline \multicolumn{8}{|c|}{ Comunidad Nueva Primavera } \\
\hline Edad (años) & $n$ & Portadores $\mathrm{n}(\%)$ & ) No portadores $\mathrm{n}(\%)$ & RMP & IC & $x^{2}$ & valor $p$ \\
\hline 1 a 15 & 10 & $6(60)$ & $4(40)$ & 7.87 & 4.03-15.35 & 41.08 & $<0.00001^{\star}$ \\
\hline 16 a 30 & 6 & $1(16)$ & $5(83.3)$ & 1 & & & \\
\hline 31 a 55 & 16 & $4(25)$ & $12(75)$ & 1.75 & $0.86-3.52$ & 2.48 & 0.114 \\
\hline 56 a 80 & 11 & $4(36.3)$ & 7 (63.6) & 2.95 & $1.50-5.78$ & 10.39 & $0.0012^{\star}$ \\
\hline Total & 43 & 15 & 28 & & & & \\
\hline
\end{tabular}


En la tabla 3 se muestra la distribución de la población con exudados faríngeos según la edad. Hubo una mayor participación de individuos pertenecientes a las edades comprendidas entre 1-15 años y 31-55 años para las tres localidades. Se realizó la prueba de X2 de tendencia lineal para las tres localidades. Para El Sabinito se encontraron diferencias estadísticamente significativas $(\mathrm{p}=0.001)$ entre el grupo de edades comprendidas entre 1-15 años y su condición de portadores, además con un riesgo 2.5 veces mayor que el resto de los grupos de edad. Para Nuevo Aquismón se encontraron diferencias estadísticamente significativas $(\mathrm{p}=$ $<0.00001)$ en el grupo de edades comprendidas de 16-30 años y en el grupo de 56-80 años ( $\mathrm{p}=0.002)$. En Nueva Primavera también se encontraron diferencias estadísticamente significativas en los grupos de edades comprendidos entre 1-15 años ( $\mathrm{p}=$ $<0.00001)$ y una RMP de 7.87 ( $\mathrm{IC}=4.03-15.35$ ). También en el grupo de 56-80 años se encontraron diferencias estadísticamente significativas $(\mathrm{p}=0.0012)$.

Se realizó un análisis entre las tres comunidades y la condición de ser portadores de BPP totales encontrándose que para la localidad de el Sabinito hay un RMP de 2.5 (IC=1.44-4.54) con un valor de $(\mathrm{p}=0.001)($ Tabla 4$)$.

Tabla 4. Comparación de portadores y probabilidad de riesgo entre las tres comunidades.

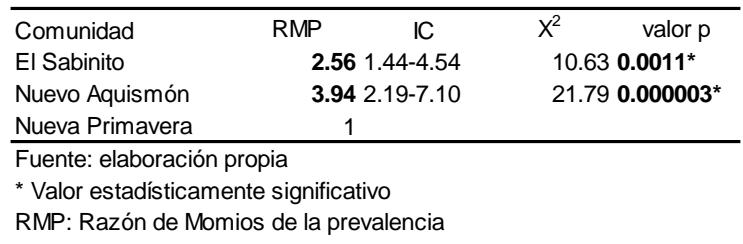

También se estableció como factor de riesgo al hacinamiento de los portadores de BPP y se pudo observar que las tres localidades presentan altos índices de este. En el Sabinito el $74 \%$ de los portadores de BPP viven en hogares hacinados, en Nueva Primavera el $44 \%$ y en Nuevo Aquismón el $79 \%$. De estas tres localidades solo 2 resultaron con diferencias significativas, El Sabinito $(\mathrm{p}=0.0007)$ y Nuevo Aquismón $(\mathrm{p}=0.001)$, además esta localidad resultó con una $\mathrm{RMP}=3.94(\mathrm{IC}=2.19-7.10)$ respecto al hacinamiento y los portadores de BPP (Tabla 5).

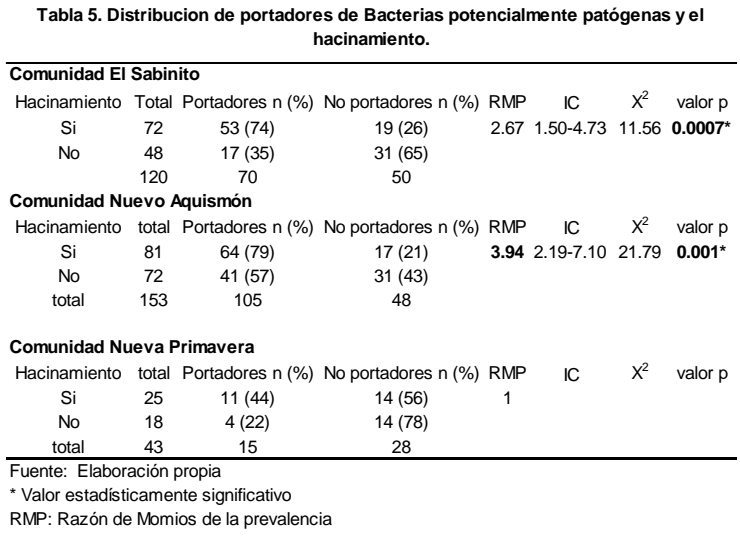

\section{Discusión}

Las prevalencias encontradas de IRA en las localidades son una aproximación preliminar de la situación de salud en comunidades vulnerables. Las diferencias encontradas en El Sabinito con respecto a las otras localidades son un indicio de que muy probablemente hay subregistros en los diagnósticos, debido en parte a la falta de infraestructura médica, falta de personal médico o las distancias para visitar uno. Nuevo Aquismón y Nueva Primavera son localidades que carecen de lo anterior, y las hace comunidades muy vulnerables en cuestión de salud. De acuerdo con el grupo de edades resultó que en la localidad de Nueva Primavera hay un riesgo de casi 8 veces más de ser portador de BPP entre 1-15 años con respecto a las demás localidades, siendo este grupo el que representa mayor vulnerabilidad a IRA, sobre todo en los menores de 5 años.

Por otra parte, las BPP representan una causa común de infección respiratoria; sin embargo, los principales géneros involucrados varían dependiendo de los estudios, sobre todo en función del tipo de cuadro respiratorio y del grupo etario involucrado (Hoeprich, 1994).

De acuerdo con los BPP identificados los más frecuentes fueron: Streptococcus pyogenes en Nuevo Aquismón, éste es el primer agente bacteriano que ocasiona faringitis, estas infecciones ocurren durante todo el año sin embargo tienen picos de incidencia en otoño y primavera, es importante diferenciar el tipo de faringitis que cursa un paciente debido a las complicaciones que puede haber ya que puede ocasionar desde otitis, sinusitis hasta escarlatina y fiebre reumática (Macedo, Mateos, 2008). 
Otro BPP encontrado fue Staphylococcus aureus (en Nuevo Aquismón y El Sabinito), patógeno capaz de causar enfermedades de amplio espectro como infecciones menores de la piel e infecciones invasoras serias como: bacteriemia, infecciones del sistema nervioso central, osteomielitis, infecciones del tracto respiratorio, infecciones del tracto urinario y el síndrome de choque tóxico, así como infecciones gastrointestinales (Cervantes, García, 2014) y por esto debe considerarse un patógeno potencial en todas las circunstancias. También ha sido implicado en la neumonía, con cifras elevadas de morbilidad y mortalidad (González, Rubio, Romero, 1999). De acuerdo con un estudio realizado en Buenos Aires sobre la distribución estacional de Staphylococcus aureus se observó que los aislamientos realizados de adultos predominaron en los meses de verano y otoño (Verón, et al., 2012). Debido a esto sería también de suma importancia hacer estudios longitudinales que permitieran encontrar los picos máximos de estos patógenos y compararlos entre otras comunidades con alta variabilidad climática.

Los portadores de este patógeno han sido estudiados en pacientes sintomáticos; así como también en individuos sanos (Corbella, et al., 1997) habiéndose reportado tasas que varían de un $18 \%$ a un $55 \%$ (Chow, Yu, 1998). Estas diferencias o variaciones en las tasas pueden corresponder a diferencias en el estudio de poblaciones, técnicas de muestreo y preservación de muestras, así como criterios de inclusión o identificación del estado del portador (López, et al., 2014) (Corbella, et al., 1997).

Se plantea que los resultados de las investigaciones epidemiológicas están influidos por la estación en que se realizan, la edad de la población estudiada y los métodos diagnósticos empleados, en la mayoría de los estudios se ha encontrado que los virus del resfriado común causan alrededor del $25 \%$ de los casos de faringitis y que otros virus son responsables de entre el 10-15\% (Chow, Yu, 1998).

De acuerdo con los factores de riesgo encontrados en este trabajo el hacinamiento resultó una condición importante para ser portador de BPP (El Sabinito y Nuevo Aquismón). Los individuos con un índice alto de hacinamiento tienen más probabilidad de desarrollar enfermedades infecciosas, así como mayor probabilidad de contagio de BPP, esto se demuestra en un estudio realizado en Suiza en donde en la población adulta e infantil estudiada se encontró que para S. pneumoniae el hacinamiento constituye un factor de riesgo (Gunnarsson, Holm, Söderström, 2000). Sería muy importante poder establecer dentro de las localidades de estudio la BPP más frecuente y su asociación con el hacinamiento, esto permitiría diferenciar las medidas preventivas o de diagnóstico oportuno muy particular y específico.

\section{Limitaciones del trabajo.}

Dado el papel fundamental que juegan las condiciones climáticas en los ciclos de vida de los patógenos identificados, una de las principales limitantes del estudio es haber realizado un único muestreo faríngeo y solamente en los meses más fríos y húmedos del año. Se considera que un estudio longitudinal hubiera arrojado información de mucho valor para la investigación y futuros programas de prevención y control de las IRA.

\section{Conclusiones}

A partir de estos hallazgos es importante señalar que los individuos que participaron en el presente estudio viven en situaciones que vulneran su salud, por ejemplo, son localidades con rezago educativo alto, con población indígena, con poca o nula atención médica local, pobreza extrema, condiciones de vivienda precarias, entre otras. Este trabajo aporta bases para poder identificar otros factores de riesgo, incluyendo la variabilidad climática, para poder ofrecer un panorama más completo de la situación con las enfermedades respiratorias y su prevención. Además, se podrían ofrecer planes o estrategias específicas y contextualizadas para cada localidad que atendieran a esta problemática de BPP e IRA.

Es importante tener en cuenta que la vinculación con otras instituciones es primordial para que este tipo de estudios se repliquen en otras comunidades con alta vulnerabilidad, no solo en el estado sino en otras partes de la república y así se puedan generar mapas de riesgo que permitan tomar decisiones en tiempo real.

\section{Agradecimientos}

Agradecemos al fondo sectorial de investigación ambiental de la Secretaría del Medio Ambiente y Recursos Naturales (SEMARNAT)_ Consejo Nacional de Ciencia y Tecnología (CONACYT) 2014-2017 en México por el financiamiento de este trabajo. 


\section{Bibliografía}

Álvarez, JP., Roas R, Maitín M. (2008). Prevalencia de bacterias potencialmente patógenas aisladas en exudado faríngeo de preescolares sanos de Barquisimeto. Boletín médico de posgrado. UCLA. Venezuela, 25 (9):1-10.

Castellanos, G M., et al. (2002). Portadores nasofaríngeos de bacterias potencialmente patógenas en pre-escolares de Maracaibo (2000 - 2001). Kasmera. 30(1): 17 - 32.

Cervantes G., R. García, P. Salazar. (2014) Características generales del Staphylococcus aureus. Revista Latinoamericana de patología clínica. 61(1):28-40.

Chow, J.; Yu, V. (1998). Staphylococcus aureus Nasal Carriage in Hemodialysis Patients. Its Role in Infection and Approaches to Prophylaxis. Arch. Intern. Med. 149:1258-1262.

Corbella, X.; Domínguez, M.; Pujol, M.; Ayats, J.; Sendra, M.; Pallares, J.; Ariza, J.; Gudiol, F. (1997). Staphylococcus aureus Nasal Carriage as a Marker for Subsequent Staphylococcal infections in Intensive Care Unit Patients. Eur. J. Clin. Microbiol. Infect. Dis.16:351-357.

Dirección General de Epidemiología. (2012). Boletín de infección de vías respiratorias agudas. Recuperado de: https://www.gob.mx/salud/acciones-yprogramas/direccion-general-de-epidemiologiainformes-semanales-para-la-vigilanciaepidemiologica-de-infecciones-respiratorias-agudas2017

Domínguez-Cortinas, G. (2013). Salud y cambio climático. Plan Estatal de Acción ante el cambio climático para San Luis Potosí.

Encuesta Nacional de Salud y Nutrición. (2012). Evidencia para la política pública en salud. Infecciones respiratorias agudas en niños: inmunizar a tiempo y educar la respuesta. México. Recuperado de: http://ensanut.insp.mx/doctos/analiticos/IRA_ninos.p df

Ferreira-Guerrero E, Báez-Saldaña R, Trejo-Valdivia B, Ferreyra-Reyes L, Delgado-Sánchez G, ChiliánHerrera OL, Mendoza-Alvarado LR, García-García L. (2013). Infecciones respiratorias agudas en niños y signos de alarma identificados por padres y cuidadores en México. Salud Publica Mex (55) supl 2:307-313.

Fuentes Y, et al. (2002). Colonización faríngea por bacterias potencialmente patógenas en niños sanos de una escuela primaria. Rev Cubana Med Trop v.61 n.1 Ciudad de la Habana ene.-abr. 2009. Disponible en: http://bvs.sldcu/revistas/mtr/vol61_1_09/mtr07109.ht $\mathrm{m}$

González C, Rubio M, Romero-Vivas J. (1999). Bacteremic pneumonia due to Staphylococcus aureus: a comparison of disease caused by methicillin-resistant and methicillin-sensitive organisms. Clin Infect Dis. 29:1171-7.

Gunnarsson RK, Holm SE, Söderström M. (2000). The prevalence of potential pathogenic bacteria in nasopharyngeal samples from healthy children and adults. Fam Pract. 17(2):150-55

Hoeprich P, Colin M, Ronald A. (1994). Bacterial pneumonias. En Infectious Diseases, EUA. Edit Lippincott.

Koneman EW, Allen SD, Dowel VR, Janda WM, Sommers HM, Winn Wc. (1998). Cocos Gram positivos. En diagnóstico microbiológico. 3ra. Edición. Editorial Médica Panamericana, 412-49.

Lieberman D, Shleyfer E, Castel H, Ferry A, HarmanBoehm Delgado, Peled J. (2006). Nasopharyngeal versus oropharyngeal sampling for isolation of potential respiratory pathogens in adults. $J$ Clin Microbiol, 44(2):525-28.

Macedo M, S. Mateos. SF. Infecciones respiratorias, en temas de bacteriología y virología médica. Recuperado de: http://www.higiene.edu.uy/cefa/2008/Infeccionesrespi ratorias.pdf

Neil W Schluger. (2010). The Acute Respiratory Infections Atlas. World Lung Foundation, New York, EUA. Pp 123.

Neto AS, Lavado P, Flores P, Días R, Pessanha MA, Sousa E, et al. (2003). Risk factors for the nasopharyngeal carriage of respiratory pathogens by Portuguese children: phenotype and antimicrobial susceptibility of Haemophilus influenzae and Streptococcus pneumoniae. Microb Drug Resist, 9(1):99-108

Organización Mundial de la Salud. (2018). Nota descriptiva. Recuperado de: http://www.who.int/es/news-room/factsheets/detail/the-top-10-causes-of-death

Secretaría de Salud (SSA). Manual de enfermedades respiratorias agudas. México (2012). Prevención, Diagnóstico y Tratamiento. (1): 1-115 [consultado en febrero 2018]. 
Infecciones respiratorias agudas, bacterias patógenas potenciales, factores de riesgo.

Somogyi T, Alfaro W, Herrera M, Herrera J. (1998). Infecciones del tracto respiratorio: etiología bacteriana y viral en una población pediátrica Rev. Méd. Hosp. Nac. Niños. Costa Rica, 33(2) n.1-2.

Tapia Conyer R, Sarti E, Kuri P, Ruiz-Matus C, Velázquez O, et al. (2006). Infecciones Respiratorias Agudas. En: Roberto Tapia Conyer, editor. El Manual de Salud Pública. México: Intersistemas, (6): 299-322.

Verón, María T.; Ojeda, María G.; Avino, Fabián; Spelzzini, Anabella; Barboza, Ana L.; Petrozzino, Yesica. (2012). Incidencia y distribución estacional de Staphylococcus aureus resistente a la meticilina en pacientes adultos ambulatorios en una clínica de la provincia de Buenos Aires: período 2006 - 2011. Revista Argentina de Microbiología. Buenos Aires Argentina, 44 (4): 306-311.

Walker CL, Rudan I, Liu L, Nair H, Theodoratou E, Bhutta ZA, et al. (2013). Global burden of childhood pneumonia and diarrhea. Lancet. EUA. 381:1405-16. 